The Congress on Research in Dance (CORD) is an interdisciplinary organization with an open. international membership. Its purposes are 1) to encourage research in all aspects of dance, including its related fields; 2) to foster the exchange of ideas, resources, and methodology through publications, international and regional conferences, and workshops; 3) to promote the accessibility of research materials.

CORD is a non-profit, tax-exempt organization. Copies of the CORD financial and operating report, filed with the State of New York, are available upon written request from the New York State Board of Social Welfare, Charities Registration Section, Office Tower, Empire State Plaza, Albany, NY 12242.

Members of CORD have voting privileges, reduced rates at conferences, special discounts on publications, and receive two journals, two newsletters, and two bulletins each membership year, which extends from January 1 to December 31 .

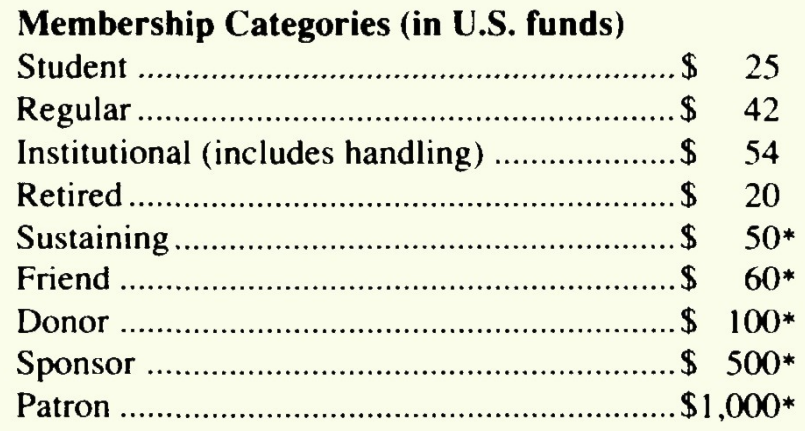

*amounts over $\$ 42$ may be considered a tax-deductible contribution

Members and institutions outside North America (Mexico, Canada, and U.S.A.)-add \$10 for airmail shipping and handling. All membership fees must be paid in U.S. funds.

\section{Executive Committee}

Sondra Fraleigh (President), Ken Aldrich (Past President), Helen Chadima (Treasurer), Judith Alter (Recording Secretary), Penelope Hanstein (Corresponding Secretary).

\section{Board of Directors}

1988-1991-Steven Chatfield, Janet Descutner, Ilene Fox, Barbara Ellen Loeb, Raphael Miller; 1989-1992-Barbara Barofsky, Judy Gantz, Nancy Brooks Schmitz, Susan Spalding; 1990-1993-Dorris Avner, Mary Bopp, Tony Pearce, Andrea Siedel.

\section{Newsletter Editor}

Jacqueline Nelson-Paunil

\section{Bulletin Editor}

Penelope Hanstein

\section{Office Administrator}

Kista Tucker

\section{Standing Committees}

Bylaws/Guidelines: Janet Descutner (chair), Judy Gantz, Raphael Miller, J.D.S. Armstrong (consultant).

Editorial Board: Steven Chatfield (chair), Gigi Berardi, Joan Erdman, Susan Foster, Barbara Palfy, Judy Van Zile.

Finance: Karen Bradley (chair), Susan Cashion, Helen Chadima, Steven Chatfield, Judy Gantz, Barbara Loeb, Raphael Miller.

Annual Conference: Nancy Brooks Schmitz (chair), Ray Cook, Joan Erdman, Sondra Fraleigh, Susan Spalding.

Elections: Janet Descutner (chair), Raphael Miller.

Regional Conferences: Steven Chatfield (chair), Karen Bradley, Joan Erdman, Penelope Hanstein.

New York Office Support: Ilene Fox (chair), Ken Aldrich, Karen Bradley.

Special thanks for editorial assistance on this issue to Marcia Sakamoto Wong.

Computer layout consultant: Dennis Ladd, University of Hawaii.

Cover design: Bharata Natyam hand gesture. Illustration by Joy Hakoda, University of Hawaii. 